Els N. Dumoulin, Stephanie Van Biervliet, Martine De Vos, Jonas Himpe, Marijn M. Speeckaert and Joris R. Delanghe*

\title{
Faecal leukocyte esterase activity is an alternative biomarker in inflammatory bowel disease
}

DOI $10.1515 / \mathrm{cclm}-2015-0040$

Received January 13, 2015; accepted March 26, 2015; previously published online April 17, 2015

\section{Abstract}

Background: Leukocyte cytosolic proteins (e.g., calprotectin) are emerging biomarkers for inflammatory bowel disease. Leukocyte aryl esterase activity has been commonly used for sensitive detection of leukocytes in human body fluids such as urine. Urine test strip results are generally reported in categories. As automated strip readers allow quantitative data to be reported, sensitive quantitative detection of leukocytes in body fluids has become possible. Here, we explored the use of leukocyte esterase as a potential alternative faecal biomarker for inflammatory bowel disease.

Methods: We evaluated leukocyte esterase activity in faecal extracts and compared Cobas u 411 (Roche) quantitative reflectance data with calprotectin concentration for 107 routine samples. Stability of leukocyte esterase for trypsin digestion was carried out by adding trypsin to the extract. Incubation occurred at $37^{\circ} \mathrm{C}$ for $24 \mathrm{~h}$ or $48 \mathrm{~h}$.

Results: Reproducibility of the reflectance signal was good (within-run imprecision: 6.1\%; between-run imprecision: $6.2 \%$ ). Results were linear in the range $10^{3}-10^{6} \mathrm{WBC} / 100 \mathrm{mg}$ faeces. The lower limit of detection was $4 \mathrm{WBC} / \mu \mathrm{L}$ and the lower limit of quantification was $5 \mathrm{WBC} / \mu \mathrm{L}$. Stability of LE activity in stool and faecal matrix was good. An adequate correlation was obtained between leukocyte esterase activity and the faecal calprotectin concentration:

*Corresponding author: Prof. Dr. Joris R. Delanghe, Laboratory of Clinical Biology, Department of Clinical Chemistry, Microbiology and Immunology, Ghent University Hospital, De Pintelaan, 185 (2P8), 9000 Ghent, Belgium, Phone: +32 933229 56, Fax: +32 933249 85, E-mail: joris.delanghe@ugent.be

Els N. Dumoulin and Jonas Himpe: Department of Clinical Chemistry, Microbiology and Immunology, Ghent University Hospital, Ghent, Belgium

Stephanie Van Biervliet: Department of Pediatric Gastroenterology and Nutrition, Ghent University Hospital, Ghent, Belgium

Martine De Vos and Marijn M. Speeckaert: Department of Internal Medicine, Ghent University Hospital, Ghent, Belgium $\log (\mathrm{y})=4.28+0.29 \log (\mathrm{x})$. In vitro experiments monitored the digestion of leukocyte esterase and faecal calprotectin. Leukocyte esterase activity was significantly less affected by trypsin activity than calprotectin immunoreactivity.

Conclusions: Quantitative leukocyte esterase activity of faecal extracts provides information about the leukocyte count in the gut lumen. Leukocyte esterase is a promising and affordable alternative biomarker for monitoring inflammatory bowel disease.

Keywords: calprotectin; faeces; inflammatory bowel disease; leukocyte esterase; trypsin.

\section{Introduction}

Crohn's disease (CD) and ulcerative colitis, collectively known as inflammatory bowel disease (IBD), are chronic illnesses of the gastrointestinal tract characterised by a recruitment of leukocytes in the intestinal wall $[1,2]$. In IBD, activated leukocytes (mostly polymorphonuclear cells) infiltrate the mucosa and appear in faeces due to shedding in the intestinal lumen $[3,4]$. Faeces is in direct contact with this inflammed intestinal mucosa, thus making stool is an obvious place for biomarker discovery in IBD [5]. High faecal neutrophil levels have been detected in IBD and may be used as markers of disease activity $[6,7]$.

At the time being, the two most commonly used faecal biomarkers for IBD are calprotectin and lactoferrin [8]. Calprotectin is a $36 \mathrm{kDa}$ protein, accounting for $60 \%$ of soluble proteins in the cytosol of neutrophils. Calprotectin is a marker of local inflammation in which the concentration reflects the neutrophil cell infiltrate in the intestinal mucosa $[9,10]$. An increased value of faecal calprotectin leads to the identification of patients with suspicion of IBD and in need of an endoscopy [9, 11]. Lactoferrin is an ironbinding glycoprotein expressed by activated neutrophils. It is released by the injured tissue during inflammation. Other faecal neutrophil-derived proteins (e.g., lysozyme, myeloperoxidase, granulocyte elastase, S100A12) have been investigated in IBD with less success [8]. Calprotectin, lactoferrin and other proteins are produced in significant 
amounts by inflammatory cells and these biomarkers correlate well with colorectal and intestinal inflammation $[12$, 13]. The faecal excretion of Indium 111-labelled leukocytes is still the golden standard faecal marker of inflammation. Unfortunately, neutrophil counting in faecal specimens is hampered by pre-analytical issues: the sample should be examined within a few hours of its collection [7].

Leukocyte esterases (LE) are cytoplasmatic enzymes present in polymorphonuclear cells. LE activity has been used for decades to detect the presence of white blood cells (WBC) in human body fluids (e.g., urine, peritoneal fluid). These esterases cleave a derivatised pyrazole amino acid ester to liberate derivatised hydroxy pyrazole. This pyrazole can react with a diazonium salt to produce a purple colour, which allows a simple and affordable quantification [14]. In 1993, Brouwer proposed semiquantitative LE analysis of faecal extracts for the monitoring of IBD. A strong correlation between faecal lysozyme concentrations and faecal LE activity was found [15]. Modern automated urine test strip analysers offer the possibility to obtain reflectance readings meaning that test results no longer need to be expressed semiquantitatively in an ordinal scale. The reflectance value (\%) is inversely related to the concentration of the analyte in the sample. Access to the instrument's raw data theoretically allows a higher analytical sensitivity and a better precision, as also shown by Penders et al. [16].

Trypsin (EC 3.4.21.4) is an endopeptidase produced in abundant amounts by the human pancreas and found in the intestinal lumen where it hydrolyses several proteins. Following release of trypsinogen, the enzyme reaches its highest activity in the proximal part of the duodenum. During transit, activity of proteases decreases $[17,18]$. As calprotectin contains 14 residues which may act as potential cleavage sites for trypsin, (partial) digestion of calprotectin by trypsin can be postulated [19]. Digestion of LE by trypsin has not yet been described.

In this study, we first wanted to explore the possibilities of faecal LE activity as a alternative biomarker for IBD, which is of major clinical importance. Additionally, we wanted to evaluate the proteolytic effect of trypsin on the faecal LE concentration.

\section{Materials and methods}

\section{Extract preparation}

Faecal extracts were prepared with the faecal sample preparation kit (Roche diagnostics, Mannheim, Germany) according to the manufacturer's instruction. After adding a standardised amount of faeces (100 mg) to a tube, $5 \mathrm{~mL}$ of EliA Calprotectin Extraction Buffer (Thermo Scientific, Uppsala, Sweden) was added and the sample was vortexed. After centrifugation (at $1200 \mathrm{~g}$ for $10 \mathrm{~min}$ ) the supernatant was used as a faecal extract in our experiments. 107 routine faecal samples from outpatients consulting the Department of GastroEnterology were used (some patients provided more than one sample with several weeks between every sample). All samples were completely processed within 2-4 $\mathrm{h}$ after arrival. The use of these samples was approved by the Ethical Committee.

\section{Method evaluation}

Test strip analysis was carried out using Combur Test M strips on the Cobas u 411 analyser (Roche diagnostics, Mannheim, Germany). The strips include reagent pads for ordinal scale reporting of relative density, $\mathrm{pH}, \mathrm{LE}$, nitrite, protein, glucose, ketones, urobilinogen, bilirubin, and haemoglobin/myoglobin. The intensity of the reaction colour of the test pad is detected by measuring the percentage of light reflected from the surface of the test pad. The reflectance value, expressed as a percentage is inversely related to the concentration of the analyte in the sample. The following equation by Penders et al. was used to estimate the number of WBC in faecal extract: $y=83.7-$ $15.4 \log (\mathrm{x})$ where $\mathrm{y}$ is the reflectance in $\%$ an $\mathrm{x}$ the number of WBC $\left(10^{6}\right.$ cells/L) [16]. First an ideal dilution was chosen for experiments and $20 \mu \mathrm{L}$ was pipetted onto the LE reagent pad. Next, within-run imprecision, between-run imprecision, linearity, stability, limit of detection, and limit of quantification were assessed for LE activity in faecal extracts. LE activity was compared to the calprotectin levels in the faecal extract and regression analysis was performed. Calprotectin was measured on the Phadia ImmunoCAP 250 analyser (Thermo Scientific, Uppsala, Sweden) according to the manufacturer's instructions. Results were expressed in $\mathrm{mg} / \mathrm{kg}$. The limit of detection of the method is $15 \mathrm{mg} / \mathrm{kg}$ [20].

\section{Proteolysis by trypsin}

Trypsin activity was determined using N $\alpha$-Benzoyl-L-arginine 4-nitroanilide hydrochloride (BAPNA, Sigma Aldrich, St. Louis, MI, USA) as a substrate for trypsin (Sigma Aldrich) in a colorimetric method. The amount of 4-nitroaniline formed was subsequently measured by the increase in absorbance at $405 \mathrm{~nm}\left(25^{\circ} \mathrm{C}\right)$. A triethanolamine solution (TEA $0.2 \mathrm{~mol} / \mathrm{L}$, Sigma Aldrich; $\mathrm{CaCl}_{2} 0.02 \mathrm{~mol} / \mathrm{L}$, Sarstedt, Nümbrecht, Germany) adjusted to $\mathrm{pH} 7.8$ was used as buffer. Results were expressed in BAPNA units (U/L). To evaluate the stability of LE activity, trypsin digestion was carried out in vitro by adding trypsin (Sigma Aldrich) solution (1 mg/mL, $1 \mathrm{mM} \mathrm{HCl}$ ). Incubation was performed at $37^{\circ} \mathrm{C}$. Samples were analysed for calprotectin and LE activity after $24 \mathrm{~h}$ and $48 \mathrm{~h}$ of incubation.

\section{Statistics}

All calculations were made in Excel 2010 and MedCalc (version 11.6.1.0, Mariakerke, Belgium). Results were given as median and inter-quartile ranges (IQR). Comparisons between conditions were made using a Wilcoxon test. A value of $\mathrm{p}<0.05$ was considered 
statistically significant. Regression analysis was performed. The lower limit of detection was calculated as the mean value plus 3 standard deviations (SD) for a blank sample. The lower limit of quantification was calculated as the mean value plus 6 SD for a blank sample.

\section{Results}

\section{Method evaluation}

Pilot experiments showed that a 1:10 dilution of faecal extract (prepared according to the manufacturer's instruction) in saline was ideal for test strip analysis and for reflectance readings. $20 \mu \mathrm{L}$ was pipetted onto the reagent pad for reflectance measurements. Reproducibility of the reflectance signal was good for LE activity in faecal extract. A within-run imprecision of $6.1 \%$ was calculated after analysing a routine sample 15 times. Consequently, a between-run imprecision of $6.2 \%$ was calculated after analysing the same sample on 15 different days. The sample contained a mean number of white blood cells of $47 \times 10^{3} / 100 \mathrm{mg}$ faeces. Results were linear in the range $10^{3}-10^{6} \mathrm{WBC} / 100 \mathrm{mg}$ faeces (corresponding to faecal calprotectin concentration ranges from \pm 1 to $\pm 1000 \mathrm{mg} / \mathrm{kg}$ ). Results are shown in Figure 1. Calprotectin levels shown are theoretically as a serial dilution was performed. The lower limit of detection for leukocytes was $4 \mathrm{WBC} / \mu \mathrm{L}$ and the lower limit of quantification was $5 \mathrm{WBC} / \mu \mathrm{L}$ (respectively corresponding to 2019 en $2688 \mathrm{WBC} / 100 \mathrm{mg}$ faeces). Data of the method evaluation are summarised in Table 1. To evaluate the stability of LE activity, 14 samples were processed and faecal matrix was placed at room temperature

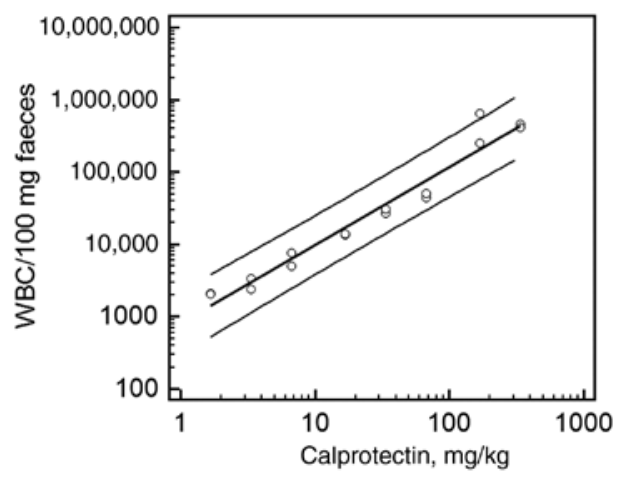

Figure 1: Linearity between calprotectin levels $(\mathrm{x}, \mathrm{mg} / \mathrm{kg})$ and leukocyte esterase (LE) activity expressed as the number of white blood cells in $100 \mathrm{mg}$ faeces (y, WBC/100 mg faeces).

Results were linear in the range $10^{3}-10^{6} \mathrm{WBC} / 100 \mathrm{mg}$ faeces (corresponding to calprotectin concentration ranges from \pm 1 to \pm 1000 $\mathrm{mg} / \mathrm{kg}$ ). WBC, white blood cells.
Table 1: Method evaluation of leukocyte esterase (LE) activity in faecal extracts. Within-run imprecision $\left(C_{w r}\right)$, between-run imprecision $\left(C_{b r}\right)$, limit of detection (LOD), limit of quantification (LOQ) were assessed. Regression analysis was performed for LE activity (y) and calprotectin levels $(x)$ in faecal extracts for 46 samples out of 107.

\begin{tabular}{ll}
\hline $\begin{array}{l}\text { Performance characteristics } \\
\text { of LE activity in faecal extracts }\end{array}$ & Results \\
\hline$C_{\mathrm{wr}}$ & $6.1 \%$ \\
$\mathrm{C}_{\mathrm{br}}$ & $6.2 \%$ \\
LOD & $4 \mathrm{WBC} / \mu \mathrm{L}$ \\
& $2019 \mathrm{WBC} / 100 \mathrm{mg}$ faeces \\
LOQ & $5 \mathrm{WBC} / \mu \mathrm{L}$ \\
& $2688 \mathrm{WBC} / 100 \mathrm{mg}$ faeces \\
Linearity & $10^{3}-10^{6} \mathrm{WBC} / 100 \mathrm{mg}$ faeces \\
Regression analysis & $\log (\mathrm{y})=4.28+0.29 \log (\mathrm{x})$ \\
& $\mathrm{r}=0.27$ \\
\hline
\end{tabular}

$\mathrm{C}_{\mathrm{w}}$, within-run imprecision; $\mathrm{C}_{\mathrm{br}}$, between-run imprecision; $L E$, leukocyte esterase; LOD, limit of detection; LOQ, limit of quantification; $r$, correlation coefficient; WBC, white blood cells.

for $24 \mathrm{~h}$. A mean decline of $1.52 \%$ was seen after $24 \mathrm{~h}$. Next, four stool samples were extracted again after $24 \mathrm{~h}$ while kept at $4{ }^{\circ} \mathrm{C}$, a difference of respectively $1.6 \%, 3.3 \%$, $0.0 \%$ and $0.9 \%$ was seen. As mentioned above, LE activity between-run imprecision was evaluated over 15 different days. These 15 aliquots were frozen at $-20^{\circ} \mathrm{C}$ and betweenrun imprecision is therefore a measurement for stability at $-20^{\circ} \mathrm{C}$.

Next, in our method evaluation we compared faecal calprotectin values and LE activity for 107 samples. After excluding samples with values below $15 \mathrm{mg} / \mathrm{kg}$ (limit of quantification for calprotectin), regression analysis was performed for 46 samples. A fair agreement was obtained between LE activity and faecal calprotectin concentration: $\log (\mathrm{y})=4.28+0.29 \log (\mathrm{x})$ with a correlation coefficient $(\mathrm{r})$ of 0.27 . LE activity is expressed as the number of white blood cells in $100 \mathrm{mg} /$ faeces [16]. Figure 2 depicts the correlation between LE activity and calprotectin concentration in faecal extracts. The arrow shows a case where proximal lesions were confirmed by endoscopy.

\section{Proteolysis by trypsin}

In vitro-experiments monitored the digestion of LE and faecal calprotectin in 10 samples. The same conditions as in our previous publication were used [19]. After adding $500 \mu \mathrm{L}$ of trypsin solution $(1 \mathrm{mg} / \mathrm{mL})$ to $500 \mu \mathrm{L}$ 1:100 diluted calprotectin in faecal matrix, proteolysis was monitored for $24 \mathrm{~h}$ and $48 \mathrm{~h}$. The median trypsin activity after adding trypsin was 57.3 U/L (IQR: 55.9-65.9 U/L). 


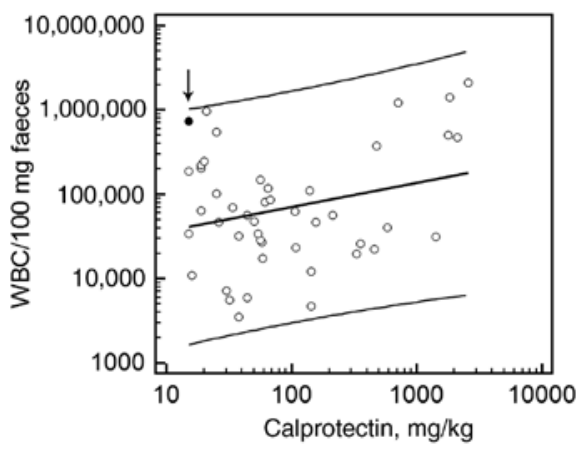

Figure 2: Regression analysis between calprotectin levels $(\mathrm{x}, \mathrm{mg} /$ $\mathrm{kg}$ ) and leukocyte esterase (LE) activity expressed as the number of white blood cells in $100 \mathrm{mg}$ faeces (y, WBC/100 mg faeces). The following regression equation was obtained: $\log (y)=4.28+0.29 \log (x)(r=0.27)$. The arrow depicts a confirmed case of IBD with proximal lesions. WBC, white blood cells.

Analogue findings for calprotectin levels were found, trypsin digestion leaded to loss of immunoreactivity and analyte stability. A median relative decrease of 59.0\% (IQR: $37.3 \%-74.3 \%$ ) was seen after $24 \mathrm{~h}$ of incubation, and a median absolute decrease of $63.5 \%$ (IQR: $32.8 \%-77.3 \%$ ) after $48 \mathrm{~h}$ of incubation. Nevertheless LE activity was less affected by trypsin activity than calprotectin immunoreactivity. After trypsin treatment, a median relative decrease of $26.5 \%$ (IQR: $13.5 \%-31.3 \%$ ) was seen after $24 \mathrm{~h}$ of incubation, and a median absolute decrease of $28.5 \%$ (IQR: $12.8 \%-32.5 \%$ ) after $48 \mathrm{~h}$ of incubation. Differences between decreases of LE activity and calprotectin levels after $24 \mathrm{~h}$ and $48 \mathrm{~h}$ of incubation were evaluated using a Wilcoxon test. Decreases were statistically significant after $24 \mathrm{~h}$ and $48 \mathrm{~h}$ of incubation (for both $\mathrm{p}=0.01$ ). Results are shown in Figure 3.

\section{Discussion}

Analysis of the test strip reflectance data allows a quantification of the analyte over a wide activity range. Using a hyperbolic calibration curve, reflectance data can be converted into quantitative WBC counts [16]. As urine test strips are inexpensive, as compared to immunochemical calprotectin testing, this could improve screening for IBD. It offers an improved, fast, reliable method that is easy to handle and usable in primary care laboratories. In our study, LE activity could be quantified in routine faecal extracts. Reproducibility of the obtained results was good (both within- and between-run imprecision were below $10 \%$ ), which is guaranteed by the constant
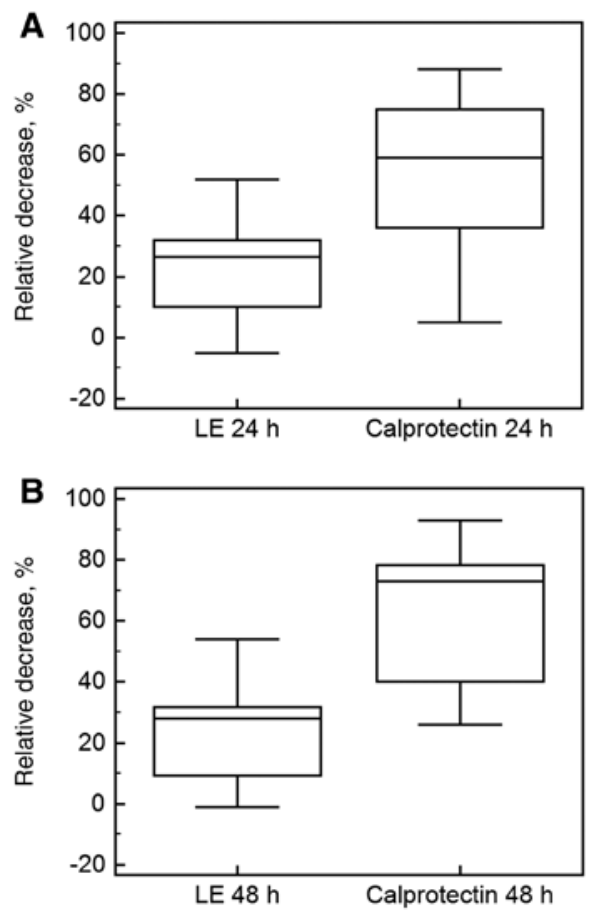

Figure 3: Distribution of relative decreases of leukocyte esterase (LE) activity and calprotectin after adding a median trypsin concentration of $57.3 \mathrm{U} / \mathrm{L}(\mathrm{IQR}: 55.9-65.9 \mathrm{U} / \mathrm{L})(\mathrm{n}=10)$ and incubation for $24 \mathrm{~h}(\mathrm{~A})$ or $48 \mathrm{~h}(\mathrm{~B})$ at $37^{\circ} \mathrm{C}$.

Differences are expressed as compared to the baseline reflectance levels (baseline=before adding trypsin). A statistically significant difference between LE activity and faecal calprotectin was found $(p=0.01)$. h, hours; LE, leukocyte esterase activity.

reaction temperature. Furthermore, the buffered extraction medium used for sample preparation adds to create an optimal environment for measuring the enzyme activity. As a result of the procedure for applying the faecal extract to the test pads on the reflectance reader, improper dipping is not a problem, neither is confusion about sample contamination caused by dipping the strip in the tube, which potentially leads to interferences.

When faecal extracts were subjected to trypsin degradation for prolonged incubation times $(24-48 \mathrm{~h})$ at $37^{\circ} \mathrm{C}$, LE proved to be more resistant against proteolysis than calprotectin. Human calprotectin shows 14 potential cleavages sites for trypsin and previous experiments showed a statistically significant breakdown of calprotectin [19]. As trypsin shows a limited stability, our findings do not imply major pre-analytical precautions for LE or calprotectin following sampling [21]. These results are in agreement with earlier data where the half-life of LE in faecal extracts was measured to be 2-3 days. In contrast, the half-life of lysozyme in faecal extracts varied between $0.5 \mathrm{~h}$ and $3.2 \mathrm{~h}[15]$. 
In this study, we further compared the LE activity quantitatively assayed on the Cobas u 411 automated strip reader with the calprotectin levels to evaluate the possible diagnostic value of faecal LE. As expected, calprotectin and LE showed an adequate correlation, as they are both markers of the number of neutrophils which have migrated through the mucosal membrane of the gut wall. In contrast to WBC count, LE is not affected by subsequent cell lyses within the gut lumen. Furthermore, dilution experiments of faecal extracts demonstrated that LE was a more sensitive biomarker than calprotectin. Test strip measurements had excellent lower limits of detection and quantification. Results were also linear in the range $10^{3}-10^{6} \mathrm{WBC} / 100 \mathrm{mg}$ faeces (corresponding to faecal calprotectin concentration ranges from \pm 1 to $\pm 1000 \mathrm{mg} / \mathrm{kg}$ ). In a previous study correlating test strip data with urinary flow results, $r$ was -0.69 between the flow cytometric WBC count and the LE reaction. The presence of esterase inhibitors in faeces and increased protein concentrations might negatively affect test results for LE [22].

The turnaround time for LE was short enough (2-4 h) not to affect the readings [23]. It is known that time intervals can influence results of automated test strip analysis, especially for leukocyte ratings. Froom et al. found that that urine testing for red and white blood cells with a dipstick is precise but unstable if testing is delayed for $24 \mathrm{~h} \mathrm{[24].} \mathrm{As} \mathrm{the} \mathrm{technique} \mathrm{is} \mathrm{not} \mathrm{time-consuming} \mathrm{and} \mathrm{well}$ known in the laboratory, the preparation of faecal matrix is only a minor addition to the workload. When samples cannot be analysed immediately, aliquots of faecal matrix can be placed at room temperature. Evaluation of stability showed that LE activity remains stable when faecal matrix is stored at room temperature (mean decline of $1.52 \%$ ) or at $-20{ }^{\circ} \mathrm{C}$ (between-run imprecision of $6.2 \%$ ). Preliminary data showed that LE activity remains stable during $24 \mathrm{~h}$ when stool is kept at $4^{\circ} \mathrm{C}$. This may be an important feature, as samples are not always brought immediately to the laboratory.

Interpretation of calprotectin values in proximal bowel disease is proven to be difficult $[25,26]$. Siponnen et al. showed a low sensitivity for calprotectin in small bowel CD. Despite active CD in the small bowel, faecal calprotectin values were low [27]. Possible explanations could be the proteolytic effect of the large amounts of trypsin released by the pancreas into the lumen of the duodenum [19]. As our experiments show that LE activity is less influenced by trypsin, it could be an alternative biomarker. Also in patients with cystic fibrosis (CF) where faecal calprotectin values are higher, possibly explained by pancreatic insufficiency, LE activity could be a valuable alternative [28]. The correlation coefficient found is rather low, which can be explained by the fact that low faecal calprotectin levels can correlate with high levels of LE activity, when proximal lesions are present. The arrow in Figure 2 depicts such a patient, where proximal lesions were confirmed by endoscopy. Summarised, LE activity cannot simply replace calprotectin, further prospective studies will be needed to assess sensitivity and specificity of the test.

In conclusion, quantitative test strip analysis provides reliable data on faecal WBC counts. LE reflectance data are promising for monitoring IBD. The faecal esterase allows non-invasive assessment of the intestinal inflammation. In comparison with immunochemical assays of specific leukocyte proteins, the LE test is about two orders of magnitude cheaper. The required technology needed for quantification is within reach of present day point of care testing. Further clinical studies will be required to define the exact role of faecal LE and the specific clinical applications in different settings of IBD patients and other patient populations. Potential clinical applications might be patients with proximal bowel disease, detecting active IBD, predicting recurrence of disease after surgery or monitoring the effects of medical therapy [8].

Acknowledgments: We wish to thank Mrs. Elke Lecocq for her technical support.

Author contributions: All the authors have accepted responsibility for the entire content of this submitted manuscript and approved submission.

Financial support: None declared.

Employment or leadership: None declared.

Honorarium: None declared.

Competing interests: The funding organisation(s) played no role in the study design; in the collection, analysis, and interpretation of data; in the writing of the report; or in the decision to submit the report for publication.

\section{References}

1. Mazlam MZ, Hodgson HJ. Peripheral blood monocyte cytokine production and acute phase response in inflammatory bowel disease. Gut 1992;33:773-8.

2. Niederau C, Backmerhoff F, Schumacher B, Niederau C. Inflammatory mediators and acute phase proteins in patients with Crohn's disease and ulcerative colitis. Hepatogastroenterology 1997;44:90-107.

3. Pepys MB, Druguet M, Klass HJ, Dash AC, Mirjah DD, Petrie A. Immunological studies in inflammatory bowel disease. Ciba Found Symp 1977;46:283-304.

4. Tibble J, Teahon K, Thjodleifsson B, Roseth A, Sigthorsson G, Bridger $\mathrm{S}$, et al. A simple method for assessing intestinal inflammation in Crohn's disease. Gut 2000;47:506-13. 
5. Bennike T, Birkelund S, Stensballe A, Andersen V. Biomarkers in inflammatory bowel diseases: current status and proteomics identification strategies. World J Gastroenterol 2014;20: 3231-44.

6. Leddin DJ, Paterson WG, DaCosta LR, Dinda PK, Depew WT, Markotich J, et al. Indium-111-labeled autologous leukocyte imaging and fecal excretion. Comparison with conventional methods of assessment of inflammatory bowel disease. Dig Dis Sci 1987;32:377-87.

7. Saverymuttu SH, Camilleri M, Rees H, Lavender JP, Hodgson HJ, Chadwick VS. Indium 111-granulocyte scanning in the assessment of disease extent and disease activity in inflammatory bowel disease. A comparison with colonoscopy, histology, and fecal indium 111-granulocyte excretion. Gastroenterology 1986;90:1121-8.

8. Angriman I, Scarpa M, D'Inca R, Basso D, Ruffolo C, Polese L, et al. Enzymes in feces: useful markers of chronic inflammatory bowel disease. Clin Chim Acta 2007;381:63-8.

9. Basso D, Zambon CF, Plebani M. Inflammatory bowel diseases: from pathogenesis to laboratory testing. Clin Chem Lab Med 2014;52:471-81.

10. Poullis A, Foster R, Mendall MA, Fagerhol MK. Emerging role of calprotectin in gastroenterology. J Gastroenterol Hepatol 2003;18:756-62.

11. van Rheenen PF, Van de Vijver E, Fidler V. Faecal calprotectin for screening of patients with suspected inflammatory bowel disease: diagnostic meta-analysis. Br Med J 2010;341:c3369.

12. Kane SV, Sandborn WJ, Rufo PA, Zholudev A, Boone J, Lyerly D, et al. Fecal lactoferrin is a sensitive and specific marker in identifying intestinal inflammation. Am J Gastroenterol 2003;98:1309-14.

13. Konikoff MR, Denson LA. Role of fecal calprotectin as a biomarker of intestinal inflammation in inflammatory bowel disease. Inflamm Bowel Dis 2006;12:524-34.

14. Moloney WC, McPherson K, Fliegelman L. Esterase activity in leukocytes demonstrated by the use of naphthol AS-D chloroacetate substrate. J Histochem Cytochem 1960;8:200-7.

15. Brouwer J. Semiquantitative determination of fecal leukocyte esterase by a dip-and-read assay. Clin Chem 1993;39:2531-2.

16. Penders J, Fiers T, Delanghe JR. Quantitative evaluation of urinalysis test strips. Clin Chem 2002;48:2236-41.
17. Layer P, Keller J. Pancreatic enzymes: secretion and luminal nutrient digestion in health and disease. J Clin Gastroenterol 1999;28:3-10.

18. Layer P, Go VL, DiMagno EP. Fate of pancreatic enzymes during small intestinal aboral transit in humans. Am J Physiol 1986;251:G475-80.

19. Dumoulin EN, Van Biervliet S, Langlois MR, Delanghe JR. Proteolysis is a confounding factor in the interpretation of faecal calprotectin. Clin Chem Lab Med 2014;53:65-71.

20. Oyaert M, Trouve C, Baert F, De Smet D, Langlois M, Vanpoucke H. Comparison of two immunoassays for measurement of faecal calprotectin in detection of inflammatory bowel disease: (pre)-analytical and diagnostic performance characteristics. Clin Chem Lab Med 2014;52:391-7.

21. Smith JS, Ediss I, Mullinger MA, Bogoch A. Fecal chymotrypsin and trypsin determinations. Can Med Assoc J 1971;104:691-4.

22. Modde H, Rodriguez S. Urin mikrobiologie. In: Colombo JP, editor. Klinisch-chemische urindiagnostik. Rotkreuz, Switzerland: Labolife Verlagsgemeinschaf, 1994:310.

23. Nagel DW, Seiler DJ. Urinalysis with the new fully automated analyzer Supertron. Eur J Clin Chem Clin Biochem 1995;33: 147-52.

24. Froom P, Bieganiec B, Ehrenrich Z, Barak M. Stability of common analytes in urine refrigerated for $24 \mathrm{~h}$ before automated analysis by test strips. Clin Chem 2000;46:1384-6.

25. Kaiser T, Langhorst J, Wittkowski H, Becker K, Friedrich AW, Ruefferet A, et al. Faecal S100A12 as a non-invasive marker distinguishing inflammatory bowel disease from irritable bowel syndrome. Gut 2007;56:1706-13.

26. Sipponen T, Karkkainen P, Savilahti E, Kolho KL, Nuutinen H, Turunen $\mathrm{U}$, et al. Correlation of faecal calprotectin and lactoferrin with an endoscopic score for Crohn's disease and histological findings. Aliment Pharmacol Ther 2008;28:1221-9.

27. Sipponen T, Haapamäki J, Savilahti E, Alfthan H, Hämäläinen E, Rautiainen $\mathrm{H}$, et al. Fecal calprotectin and S100A12 have low utility in prediction of small bowel Crohn's disease detected by wireless capsule endoscopy. Scand J Gastroenterol 2012;47:778-84.

28. Lee JM, Leach ST, Katz T, Day AS, Jaffe A, Ooi CY. Update of faecal markers of inflammation in children with cystic fibrosis. Mediators Inflamm 2012;2012:948367. 